TI 2012-30/1

Tinbergen Institute Discussion Paper

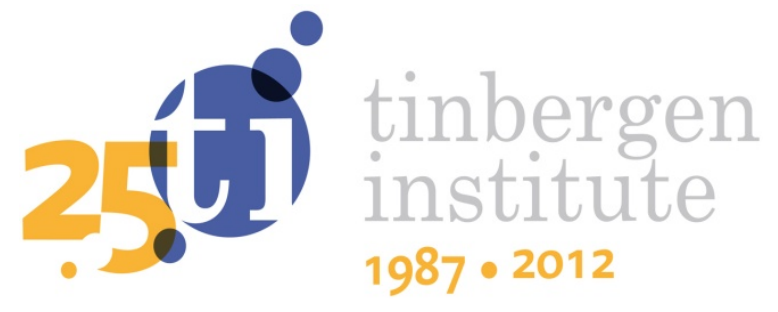

\title{
On the Measurement of Success and Satisfaction
}

René van den Brink ${ }^{1}$

Frank Steffen²

${ }^{\prime}$ Department of Econometrics, Faculty of Economics and Business Administration, VU University, and Tinbergen Institute;

2 Department of Economics, The University of Liverpool Management School. 
Tinbergen Institute is the graduate school and research institute in economics of Erasmus University Rotterdam, the University of Amsterdam and VU University Amsterdam.

More TI discussion papers can be downloaded at http://www.tinbergen.nl

Tinbergen Institute has two locations:

Tinbergen Institute Amsterdam

Gustav Mahlerplein 117

1082 MS Amsterdam

The Netherlands

Tel.: +31(0)205251600

Tinbergen Institute Rotterdam

Burg. Oudlaan 50

3062 PA Rotterdam

The Netherlands

Tel.: +31(0)10 4088900

Fax: $+31(0) 104089031$

Duisenberg school of finance is a collaboration of the Dutch financial sector and universities, with the ambition to support innovative research and offer top quality academic education in core areas of finance.

DSF research papers can be downloaded at: http://www.dsf.nl/

Duisenberg school of finance

Gustav Mahlerplein 117

1082 MS Amsterdam

The Netherlands

Tel.: +31(0)20 5258579 


\title{
On the Measurement of Success and Satisfaction ${ }^{1}$
}

\author{
René van den Brink ${ }^{2}$ \\ Frank Steffen ${ }^{3}$
}

March 19, 2012

\footnotetext{
${ }^{1}$ We would like to thank Matthew Braham, Keith Dowding, William Gehrlein, Manfred Holler, Serguie Kaniovski, Dennis Leech, Moshé Machover, Peter Morriss, and Stefan Napel for comments and discussions. Considerable parts of the research contained in this paper were already developed between 2005 and 2006 when Frank Steffen was at Tilburg University under a Marie Curie IntraEuropean Fellowship within the 6th European Community Framework Programme. He gratefully acknowledges this financial support.

${ }^{2}$ Department of Econometrics and Tinbergen Institute, VU University, De Boelelaan 1105, 1081 HV Amsterdam, The Netherlands, E-mail: jrbrink@feweb.vu.nl.

${ }^{3}$ Department of Economics, Finance and Accounting, The University of Liverpool Management School (ULMS), Chatham Street, Liverpool L69 7ZH, U.K., E-mail: steffen2@liverpool.ac.uk.
} 


\begin{abstract}
The main purpose of the present paper is to disentangle the mix-up of the notions of success and satisfaction which is prevailing in the voting power literature. We demonstrate that both notions are conceptually distinct, and discuss their relationship and measurement. We show that satisfaction contains success as one component, and that both coincide under the canonical set-up of a simultaneous decision-making mechanism as it is predominant in the voting power literature. However, we provide two examples of sequential decision-making mechanisms in order to illustrate the difference between success and satisfaction. In the context of the discussion of both notions we also address their relationship to different types of luck.
\end{abstract}

Keywords: success, satisfaction, luck, power

JEL Classification: C79, D02, D71 


\section{Introduction}

In their volume 'The Measurement of Voting Power' Felsenthal and Machover (1998) point out that the idea of the measurement of success has been part of the theory of voting power since the first scientific contribution in the field by Penrose (1946) and since then has been rediscovered by several other scholars. Despite this fact the notion of success has received relatively little attention for a number of decades as - to make use of Laruelle et al.'s (2006) terminology - it was often regarded to be "just a sort of appendix" of power. The most prominent early supporters of this view are said to be Dubey and Shapley (1979) who have proven that there exists a linear relationship between power measured by the Banzhaf (1965) measure and success measured by their 'agreement index', which is an extended version of the 'Rae (1969) index' (see, for instance, Laruelle et al. 2006). ${ }^{1}$ More contemporary supporters are Hosli and Machover (2004) who accentuate that the notion of success is 'virtually identical' with the notion of power and that both "differ only in using a different scale of measurement". Only recently, Laruelle and Valenciano (2005, 2008) and Laruelle at al. (2006) have emphasized and vindicated the relevance of success for the normative assessment of collective decision-making mechanisms and have demonstrated that the aforementioned view is misleading.

While their contribution deserves to be acknowledged, they still continue to contribute to an ongoing common confusion in the voting power literature: they appear to regard satisfaction to be a synonym for success. A careful re-examinination of the voting power literature with respect to both notions leads to the following conclusion. In the literature we can find two outcome related correspondences being applied: one which relates an actor's 'action' (or 'vote') to the collective outcome, let us call this the 'action-outcome correspondence' (AOC), and a second one which replaces 'action' by 'inclination' (or 'preference') and which we will call the 'inclination-outcome correspondence' (IOC). Both are used to define what authors claim to be success or satisfaction, respectively. Moreover, in the literature we can find a third notion being called '(individual-group) agreement' which is also used as a label for both correspondences. Note that in comparison to the other two notions, i.e., success and satisfaction, this one is 'neutral' as it leaves it unspecified whether the agreement on the individual level refers to an action or an inclination.

An overview of the combinations of notions and correspondences applied in the literature by different authors is given in Table $1 .{ }^{2}$ In addition to the information included in Table 1 the following should be noted. In this strand of literature the usual core point of

\footnotetext{
${ }^{1}$ Note that Dubey and Shapley (1979) use the notion of 'agreement' instead of success and that a proof of this relationship can also be found in Brams and Lake (1982) who apply the notion of satisfaction. The relationship between these three notions will be clarified in the course of this paper.

${ }^{2}$ While Table 1 includes the most prominent sources, we do not claim completeness.
} 
reference is Rae (1969). He refers to the IOC by speaking about what an actor 'would like to have' with respect to the collective outcome, but does not introduce a certain notion for this correspondence. Given the nature of his analysis, in our view, the most appropriate notion appears to be individual-group agreement. This notion is applied by Brams and Lake (1978) when they refer to Rae (1969). However, when referring to Rae (1969) starting with Straffin (1977) most authors in the field are careless as they (partly implicitly) claim (i) that Rae (1969) is referring to the AOC and/or (ii) that he introduced the notion of success and/or (iii) that success and/or satisfaction are appropriate labels for the IOC. Notable

Table 1: Success and Satisfaction in the Voting Power Literature

\begin{tabular}{|c|c|c|}
\hline $\begin{array}{l}\text { Correspon- } \\
\text { dence }\end{array}$ & Notion & Source \\
\hline \multirow{3}{*}{$\mathrm{AOC}$} & Success & $\begin{array}{l}\text { Laruelle and Valenciano }(2005,2008) \\
\text { Laruelle et al. (2006) }\end{array}$ \\
\hline & Satisfaction & $\begin{array}{l}\text { Brams and Lake (1978) } \\
\text { Nevison }(1978,1982) \\
\text { Straffin at al. (1982) } \\
\text { Laruelle and Valenciano }(2005,2008) \\
\text { Laruelle et al. (2006) }\end{array}$ \\
\hline & (Individual-Group) Agreement & $\begin{array}{l}\text { Straffin (1977) } \\
\text { Dubey and Shapley (1979) } \\
\text { Laruelle and Valenciano }(2005,2008)\end{array}$ \\
\hline \multirow[b]{2}{*}{ IOC } & Success & $\begin{array}{l}\text { Barry (1980) } \\
\text { Dowding (1991, 1996) } \\
\text { Grabisch and Rusinowska (2010) }\end{array}$ \\
\hline & $\begin{array}{l}\text { Satisfaction } \\
\text { (Individual-Group) Agreement }\end{array}$ & $\begin{array}{l}\text { van den Brink et al. (2011) } \\
\text { Rae (1969) } \\
\text { Felsenthal and Machover (1998) } \\
\text { Hosli and Machover (2004) }\end{array}$ \\
\hline
\end{tabular}


exceptions are, for instance, Holler (1982), Felsenthal and Machover (1998), and Hosli and Machover (2004). Finally, the contributions by Barry (1980) and Dowding (1991, 1996) deserve some comments. First, it has to be noted that both do not make any reference to Rae (1969) or any of the other authors listed in Table 1, and that Dowding's $(1991,1996)$ unique point of reference in this context is Barry (1980). Secondly, it has to be mentioned that Barry (1980), who applies the IOC and uses success as a label for this correspondence, adds the additional requirement that the actor in question, in order to be successful, must have chosen an action ('the actor has tried') before the collective outcome occurred. ${ }^{3}$

Having outlined the mix-up in the literature the obvious question of the contribution of its disentanglement arises, i.e., (i) whether this is of historical interest only, and has no consequence for an analysis, or (ii) whether there exists an outstanding problem which could be solved only after this exercise has been completed. The brief answer is that both are true. We show that (i) applies if we restrict ourselves to the canonical set-up in the voting power literature, that is simultaneous decision making, as under this set-up success and satisfaction coincide, while (ii) is correct if we deviate from this set-up, for instance, by allowing for a sequential decision-making mechanism such as the one introduced in van den Brink and Steffen $(2008,2012)$. It will turn out that satisfaction entails success as one component.

The remaining paper is organized as follows. Section 2 recapitulates the canonical set-up of a decision-making mechanism in the voting power literature and adds some further assumptions and definitions required to relax the canonical setup in the course of our analysis. In Section 3 we investigate the nature of success and satisfaction and provide general definitions of corresponding measures which are also applicable for sequential decision-making mechanisms. In Section 4 we discuss the relationship between success and satisfaction which requires us to address also the notions of power and luck. In Section 5 we illustrate our results from Section 4 by two examples of a sequential decision-making mechanism. Concluding remarks considering the impact of 'abstention' on the relationship between success and satisfaction are contained in Section 6.

\footnotetext{
${ }^{3}$ We would like to note that Barry (1980) himself defines success as the probability to be successful as defined above. A discussion of this issue can be found in Dowding (1991: 65). Moreover, note that Barry's (1980) additional requirement is always fulfilled under the canonical set-up as specified in Section 2 as under this set-up (i) abstention is not permissible and (ii) all actors have to choose their action simultaneously. The issue of 'abstention' and its implications for the relationship between success and satisfaction are addressed in Section 6.
} 


\section{Preliminaries}

A collective decision-making mechanism (DMM) $\Gamma$ consists of a decision rule and a decisionmaking procedure. A decision rule is a function which maps ordered sets of individual actions into outcomes, i.e., it states which ordered set of actions generate which outcome. A decision-making procedure provides the course of actions of the actors for a collective decision and determines the actions to be counted, i.e., which actions go into the domain of the decision rule. ${ }^{4}$

Assumption 2.1 For the purpose of this paper let us make the following assumptions regarding $\Gamma$ :

1. Proposals submitted to the decision-making body are exogenous: it is the task of the decision-making body either to accept or to reject a proposal, i.e., we have a binary outcome set $O=\{$ approval, rejection $\}$.

2. A proposal can be submitted to the decision-making body only once.

3. The decision-making body contains a finite set of actors: $N=\{1, \ldots, n\}$ with $n>1$, whose actions bring about a collective outcome of the decision-making body.

4. Each actor $i \in N$ has a binary action set: $A_{i}=\{y e s, n o\}$, where the choice of the yes-action means that $i$ supports the proposal and the choice of the no-action that $i$ rejects it. ${ }^{5}$

5. All actors choose their action simultaneously ${ }^{6}$

6. Each actor $i \in N$ has a binary inclination set: $K_{i}=\{$ approve, reject $\}$ containing $i$ 's feasible attitudes towards a proposal.

Remark 2.2 Note that we treat inclinations and preferences as synonyms. They express what actors 'want' or 'like'. Hence, they have to be distinguished from the actors' choice of action.

In the voting power literature the canonical set-up of an DMM $\Gamma$ is based on the first five assumptions of Assumptions 2.1. Such a canonical set-up is, usually, represented by a

\footnotetext{
${ }^{4}$ For further details, see van den Brink and Steffen (2008, 2012).

${ }^{5}$ This excludes the option of abstention as a tertium quid, which can have a considerable impact on the power distribution among the actors (see, for instance, Felsenthal and Machover 1997 1998, and Braham and Steffen 2002). In Section 6 we will briefly address the possibility of abstention in the context of the analysis of the present paper.

${ }^{6}$ Note that this implies that a secret but sequential decision-making procedure is permissible as well.
} 
simple voting game $(\mathrm{SVG})$, being a pair $(N, \mathcal{W})$ where $\mathcal{W}$ is a collection of subsets called 'coalitions', which satisfies the following three conditions: (i) $\emptyset \notin \mathcal{W}$, (ii) $N \in \mathcal{W}$, and (iii) if $T \in \mathcal{W}$ and $T \subseteq T^{\prime}$, then $T^{\prime} \in \mathcal{W}$ (monotonicity). A coalition $T \subseteq N$ is said to be winning or losing according to whether $T \in \mathcal{W}$ or $T \notin \mathcal{W}$. This definition implies that an SVG can also be represented by $\mathcal{W}$ only. Moreover, a coalition $T$ can be regarded as an 'index' of the actions of actors who have chosen the same action, for instance, 'yes' if $T \in \mathcal{W}$. Whether a coalition is winning or losing is determined by the decision rule being applied.

The analysis of the canonical set-up represented by an SVG is primarily based on the membership of actors in coalitions. For this reason van den Brink and Steffen (2008, 2012) have called this approach the 'membership-based approach'. They also introduced an 'action-based approach' which allows other DMM's, in particular it allows sequential decision making where it can happen that not all actors will be allowed to choose an action. This has already been taken into account for the formulation of Assumption 2.1. ${ }^{7}$ Next, we define action and inclination profiles.

Definition 2.3 Given a subset of actors $S \subseteq N$, an action profile $a=\left(a_{i}\right)_{i \in S}$ on $S$ is a non-empty ordered set of individually chosen actions $a_{i} \in A_{i}, i \in S$.

Note that it is not required that an action profile contains an action for every actor. However, all actors have an inclination as expressed in the following definition:

Definition 2.4 An inclination profile $k=\left(k_{i}\right)_{i \in N}$ is a ordered set of inclinations $k_{i} \in K_{i}$, $i \in N$.

So, an inclination profile $k$ contains one, and only one, inclination $k_{i}$ for each $i \in N$. We denote the collection of all action profiles on any $S \subseteq N$ by $\mathcal{A}^{N}$, and the collection of all action profiles containing an action of actor $i$ (i.e. the action profiles on $\{S \subseteq N \mid i \in S\}$ ) by $\mathcal{A}_{i}^{N}$. We denote the actors who are choosing an action in an action profile $a$ by $N(a)$, i.e. $N(a)=S$ for $a=\left(a_{i}\right)_{i \in S}$. Further we denote the collection of all inclination profiles on $N$ by $\mathcal{K}^{N}$.

Remark 2.5 Assumptions 1, 4 and 5 listed under Assumption 2.1 together with the assumption that agents choose a dominant strategy whenever it exists, imply that under the canonical set-up all actors will always act sincerely, i.e., they will choose an action which corresponds with their own inclinations as it is a dominant strategy for each actor to choose its own first choice (see, for instance, Laruelle and Valenciano 2008: 55). If, for instance, an actor $i$ has the inclination to 'approve' a proposal it will always choose the

\footnotetext{
${ }^{7}$ For a detailed description of the 'action-based approach' we refer to van den Brink and Steffen (2008).
} 
yes-action. From this we can infer that under the canonical set-up there is no need for a separate modeling of the actors' inclinations, assuming that an actor chooses its action according to its inclination. Hence, its representation by a bare SVG is sufficient.

Remark 2.6 Assumptions 4 and 5 of Assumption 2.1 together imply that all actors are not only allowed, but are also obliged to choose an action which goes into the domain of the decision rule.

Remark 2.6 allows us to specify Definition 2.3 for the canonical set-up as follows:

Definition 2.7 $A$ simultaneous action profile $a=\left(a_{i}\right)_{i \in N}$, is a non-empty ordered set of individually chosen actions $a_{i} \in A_{i}, i \in N$.

So, a simultaneous action profile $a$ contains one, and only one, action $a_{i}$ for each $i \in N$. What is left to be formulated at this stage are expressions denoting the probabilities that a permissible action profile $a$ and a feasible inclination profile $k$ being compatible with $a$ occurs. The latter is required as for a sequential DMM $\Gamma$ it can happen, that more than one inclination profile $k$ is compatible with a single action profile $a$ (see Section 5 ).

Definition 2.8 Consider an DMM $\Gamma$. An inclination profile $k$ is compatible with action profile a if, and only if, for all $i \in N(a)$ it holds that (i) $k_{i}=$ 'approve' if $a_{i}=$ 'yes', and (ii) $k_{i}=$ 'reject' if $a_{i}=$ 'no'. We denote by $p(a, \Gamma)$ the probability of the occurrence of an action profile $a$, and by $\tilde{p}(k, a, \Gamma)$ the probability of an inclination profile $k$ which is compatible with a.

We denote the set of all inclination vectors that are compatible with action profile $a$ by $K(a) .{ }^{8}$ We introduce the notation $k_{i} \sim a_{i}$ meaning that the inclination $k_{i}$ corresponds with the action $a_{i}$ of actor $i$ in the sense that ' $k_{i}=$ approve' if $a_{i}=$ 'yes', and ' $k_{i}=$ reject' if $a_{i}=$ 'no'. Similar, we use the notation $a_{i} \sim o, o \in O$, meaning that the action $a_{i}$ corresponds with the outcome $o$ in the sense that $a_{i}=$ 'yes' if ' $o=$ approval', and $a_{i}=$ 'no' if ' $o=$ rejection'. Finally, we use the notation $k_{i} \sim o, o \in O$, meaning that the inclination $k_{i}$ corresponds with the outcome $o$ in the sense that $k_{i}=$ 'approve' if ' $o=$ approval', and $k_{i}=$ 'reject' if ' $o=$ rejection'. ${ }^{9}$

\footnotetext{
${ }^{8} \mathrm{So}, p(\cdot, \Gamma)$ is a probability distribution over $\mathcal{A}^{N}$, while $\tilde{p}(\cdot, a, \Gamma)$ is a conditional probability distribution over $K(a)$.

${ }^{9}$ Note that it would be possible to 'code' the actions, inclinations and outcomes by 0 and 1 , in which case the correspondences $\sim$ defined above could be simply written as equalities. We chose not to do that in this paper to make clear the distinction between actions and inclinations, which is essential for the difference between success and satisfaction (and luck) later on.
} 
Remark 2.9 Note that from remarks 2.5 and 2.6 it follows immediately that under the canonical set-up there exists one, and only one, inclination profile $k$ which corresponds to an action profile $a$, i.e., $\forall a: \exists \tilde{k}: \tilde{p}(\tilde{k}, a, \Gamma)=1$, while $\forall k \neq \tilde{k}: \tilde{p}(k, a, \Gamma)=0$, since $N(a)=N$ in the canonical set-up.

\section{Success and Satisfaction}

At the outset of this section let us start with a definition of the notions of success and satisfaction. For this purpose we refer to Collins English Dictionary (2009) and the American Heritage Dictionary of the English Language (2011):

Definition 3.1 Success is 'the favourable outcome of something attempted'.

Remark 3.2 The reference to an 'attempt' in Definition 3.1 implies that success inherently requires an action. However, it is 'inclination-free' as no reference is made to whether that what was attempted was something the actor desired or not.

Definition 3.3 Satisfaction is 'the fulfilment of a desire'.

Remark 3.4 The reference to a 'desire' in Definition 3.3 implies that satisfaction inherently requires an inclination. However, it does not necessarily require an action as it is feasible that a desire can be fulfilled without having made any attempt at all. ${ }^{10}$

Thus, in general, success and satisfaction are distinct concepts. Making use of definitions 3.1 and 3.3 we can now define how success and satisfaction ought to be ascribed to an actor being a member of a decision-making body under an DMM $\Gamma$, which is characterized by the assumptions as listed under Assumption 2.1. We ascribe success to $i$ if, and only if, $i$ 's chosen action corresponds with the collective outcome.

Definition 3.5 Let $S \subseteq N$ and $a=\left(a_{i}\right)_{i \in S}$ be an action profile with $a_{i} \in A_{i}$ for all $i \in S$. Then, the success ascription to $i \in S$, given the collective outcome o $(a, \Gamma) \in O$ is given by

$$
\overline{S U C}_{i}(a, \Gamma)= \begin{cases}1 & \text { if } a_{i} \sim o(a, \Gamma) \\ 0 & \text { otherwise }\end{cases}
$$

\footnotetext{
${ }^{10}$ This can be illustrated by the following example which we owe to Matthew Braham. Assume that you have the desire to become rich, but you are not doing anything to achieve this. Instead, you are lying on the beach enjoying the sun and the fresh air. However, suddenly one of the seagulls circling over your head drops a valuable diamond which just falls into your lap. Thus, your desire has been fulfilled even you have not attempted anything to achieve this, if we assume that you were not aware of the fact that it might happen that a seagull drops a valuable diamond during the time you are lying on that beach.
} 
Remark 3.6 If the canonical set-up is represented by an SVG, success is ascribed to an actor $i$ if, and only if, $i$ is a member of a winning coalition, i.e., if, and only if, $(i \in T \in$ $\mathcal{W})$ or $(i \notin T \notin \mathcal{W}$ ) (see, for instance, Laruelle and Valenciano 2005, 2008).

We ascribe satisfaction to $i \in N$, if and only if, $i$ 's inclination $k_{i} \in k$ corresponds with the collective outcome $o \in O$, where the collective outcome results out of an action profile $a$, which is compatible with the inclination profile $k$. Note that the collective outcome $o$ is the same for all inclination profiles $k$ which are compatible with the action profile $a$.

Definition 3.7 Let $k=\left(k_{i}\right)_{i \in N}$ be an inclination profile. Then, the satisfaction ascription to $i \in N$, is given by

$$
\overline{S A T}_{i}(k, \Gamma)= \begin{cases}1 & \text { if } k_{i} \sim o(a, \Gamma) \text { for } a \in \mathcal{A}^{N} \text { with } k \in K(a) \\ 0 & \text { otherwise. }\end{cases}
$$

Based on Definitions 3.5 and 3.7 we can now formulate corresponding success and satisfaction measures. The success of an actor $i \in N$ in a decision-making body applying an DMM $\Gamma$ is measured by $i$ 's probability to be successful, i.e., by summing-up over $i$ 's weighted success ascriptions $\overline{S U C}_{i}(a, \Gamma)$ for all feasible action profiles $a \in \mathcal{A}_{i}^{N}$ where $i$ chooses an action.

Definition 3.8 The success measure SUC of a decision-making body applying an DMM $\Gamma$ is given by

$$
S U C_{i}(\Gamma)=\sum_{a \in \mathcal{A}_{i}^{N}} p(a, \Gamma) \overline{S U C}_{i}(a, \Gamma) \quad \text { for each } i \in N .
$$

The satisfaction of an actor $i \in N$ in a decision-making body applying an DMM $\Gamma$ is measured by $i$ 's probability to be satisfied, i.e., by summing-up over $i$ 's weighted satisfaction ascriptions $\overline{S A T}_{i}(k, \Gamma)$ for all feasible inclination profiles $k .{ }^{11}$

Definition 3.9 The satisfaction measure $S A T$ of a decision-making body applying an DMM $\Gamma$ is given by

$$
S A T_{i}(\Gamma)=\sum_{a \in \mathcal{A}^{N}} p(a, \Gamma) \sum_{k \in K(a)} \tilde{p}(k, a, \Gamma) \overline{S A T}_{i}(k, \Gamma) \text { for each } i \in N .
$$

\footnotetext{
${ }^{11}$ Note that van den Brink et al. (2011) are making use of a version of this measure. However, referring to Straffin et al. (1982) they were unaware of the fact that the correspondence used in Straffin et al. (1982) does not coincide with Rae's (1969).
} 
Remark 3.10 Based on definitions 3.5 to 3.9 in conjunction with remarks 2.5 and 2.6 it is straightforward to see that under the canonical set-up being applied in the voting power literature the values of both measures will always coincide. Hence, there exists no good reason for the usage of two separate measures. As the canonical set-up is, usually, represented by an SVG which relates actions to collective outcomes, but does not include any inclinations, it appears to be reasonable that in this context studies focus on the success measure and ignore the satisfaction measure. This is, in fact, what we can observe in the recent voting power literature (see, for instance, Laruelle and Valenciano 2005, 2008).

\section{Satisfaction, Success, Power, and Luck}

After having discussed the notions of success and satisfaction and its measurement in Section 3 their relationship still remains to be clarified. For the purpose of this exercise let us begin with a brief discussion of Barry's (1980) well-known equation 'success $=$ power + luck' ${ }^{12}$ In Section 1 we already adressed Barry's (1980) definition of success which according to our analysis in Section 3 is, in fact, a definition of satisfaction. However, when referring to Barry (1980) in the more recent literature authors have just replaced the IOC, which Barry (1980) originally presupposes, by the AOC (see, for instance, Laruelle and Valenciano 2005, 2008, Laruelle et al. 2006, and, referring to Laruelle and Valenciano 2005, also Rusinowska and de Swart 2006 and Grabisch and Rusinowska 2010). In a similar fashion, when referring to luck the same authors have also replaced Barry's (1980) original definition of 'luck' by an essentially different one. We will come back to this issue in the course of this section. However, before we would like to specify the notion and measurement of power which we apply for our analysis.

The notion of power in this paper is based on Braham (2008) and Morriss (1987/2002). In a social context they define power as an ability (or capacity) to effect (i.e., to 'force' or 'determine') outcomes and regard power to be a dispositional concept, i.e., power exists whether it is exercised or not. Following Braham (2008) we say that an actor $i$ has power with respect to a certain outcome if $i$ has an action (or sequence of actions) such that its performance under the stated or implied conditions will result in that outcome despite of the actual or possible resistance of at least some other actor. That is, power is a claim about what $i$ is able to do against some resistance of others irrespective of its actual occurrence.

\footnotetext{
${ }^{12}$ Note that Barry (1980) does not use the notion of 'power' in this context, but refers to decisiveness. However, by this notion he means what is usually called 'power' in the voting power literature. For a discussion of this issue see, for instance, Dowding (1991: 63-68, 1996: 52-54) or Felsenthal and Machover (1998: 41).
} 
We ascribe power to an actor in an action profile if, and only if, this actor acts in this profile and by choosing a different action from its action set is able to alter the collective outcome against some resistance of others (represented by those chosen actions of the other actors which are not in line with the 'new' action of the actor in question). In this case we say that the actor has a swing. With respect to our analysis it needs to be noted that for an DMM $\Gamma$ the notion of a swing as used in the canonical setup is no longer sufficient since an actor changing its action in a certain action profile, might lead to other actors, who did not have an action in that action profile, to get the possibility to act. On the other hand, it might be that actors who did act now cannot act anymore. As a result, it might be that by changing its action an actor might (but not necessarily does) change the outcome. Therefore, we have to distinguish between strong and weak swings (see Section 6, but also van den Brink and Steffen 2008, 2012 for the case of a sequential DMM).

Let $S \subseteq N$, and $a=\left(a_{j}\right)_{j \in S}$ be an action profile containing action $a_{j} \in A_{j}, j \in S$. Then, we say that $i$ has a strong swing if by altering its choice of action, $i$ forces, ceteris paribus, a new collective outcome. ${ }^{13}$ Given action profile $a=\left(a_{j}\right)_{j \in S}$, actor $i \in S$, and alternative action $\hat{a}_{i} \in A_{i} \backslash\left\{a_{i}\right\}$, we denote by $\mathcal{A}_{i, \hat{a}}^{a}=\left\{\tilde{a}=\left(\tilde{a}_{j}\right)_{j \in T} \in \mathcal{A}^{N} \mid T \subseteq N, i \in\right.$ $T, \tilde{a}_{i}=\hat{a}_{i}$ and $\tilde{a}_{j}=a_{j}$ for all $\left.j \in S \cap T\right\}$ the set of all action profiles that are possible after actor $i$ changes its action from $a_{i}$ to $\hat{a}_{i}$.

Definition 4.1 Let $S \subseteq N$, and $a=\left(a_{j}\right)_{j \in S}$ be an action profile containing actions $a_{j} \in$ $A_{j}, j \in S$. Then, we say that $i \in S$ has a strong swing in $S$, if there is an $\hat{a}_{i} \in A_{i} \backslash\left\{a_{i}\right\}$

\footnotetext{
${ }^{13}$ As van den Brink and Steffen (2008) point out it is important to draw attention to the interpretation of the ceteris paribus condition in this context. Its common interpretation is that the actions of all other actors remain constant. That is, if $i$ alters its action the only effect that can result out of this is a change in the collective outcome (then we say that $i$ has a swing and we ascribe power to $i$ ). While this 'all other things being equal' interpretation is appropriate for simultaneous DMMs, it no longer applies for our more general case of a sequential DMM, which may allow certain actors to exclude other actors from the decision-making as a result of their choices. If we have an action profile and we alter $i$ 's choice of action it can happen that the decision-making process requires either the exclusion of actions of other actors from the domain of the decision rule and, hence, from the action profile, or the inclusion of actions by other actors in the domain of the decision rule and, therefore, in the action profile. If such information would be ignored, we can end up with an inappropriate power ascription. In order to avoid this problem we have to go back to the idea behind the literal 'all other things being equal' interpretation of the ceteris paribus clause. The basic idea of the ceteris paribus clause is a comparison between two possible worlds: the world as it is (our initial action profile and its associated collective outcome) and the world as it would be if an action were changed (the resulting action prole and its associated collective outcome if $i$ 's choice of action were altered). In contrast to the standard interpretation of the ceteris paribus clause our analysis does not necessarily require that all other components of the action profile remain constant after we altered $i$ 's choice of action; it requires that the action profiles after the initial change by one actor are consistent with the DMM. This interpretation of the ceteris paribus clause is underlying definitions 4.1 and 4.2.
} 
such that $o(\tilde{a}, \Gamma) \neq o(a, \Gamma)$ for all $\tilde{a} \in \mathcal{A}_{i, \hat{a}}^{a}$.

Let $S \subseteq N$ and $a=\left(a_{j}\right)_{j \in S}$ be an action profile containing an action $a_{j} \in A_{j}$, $j \in S$. Then, we say that $i$ has a weak swing, if by altering its choice of action, it is, ceteris paribus, feasible that a new collective outcome emerges, but that the outcome does not change necessarily.

Definition 4.2 Let $S \subseteq N$, and $a=\left(a_{j}\right)_{j \in S}$ be an action profile containing actions $a_{j} \in$ $A_{j}, j \in S$. Then, we say that $i \in S$ has a weak swing in $S$ if there exists an $\hat{a} \in A_{i} \backslash\left\{a_{i}\right\}$ and $\tilde{a} \in \mathcal{A}_{i, \hat{a}}^{a}$ such that $o(\tilde{a}, \Gamma) \neq o(a, \Gamma)$, and also there exists an $\hat{a} \in A_{i} \backslash\left\{a_{i}\right\}$ and $\tilde{a} \in \mathcal{A}_{i, \hat{a}}^{a}$ such that $o(\tilde{a}, \Gamma)=o(a, \Gamma)$.

Remark 4.3 Note that under the canonical setup of a simultaneous DMM there exist no weak swings. Hence, all swings are strong. For a given action profile $a=\left(a_{j}\right)_{j \in N}$ actor $i \in N$ has a swing in $a$ if $o(\tilde{a}, \Gamma) \neq o(a, \Gamma)$ with $\tilde{a}=\left(\tilde{a}_{j}\right)_{j \in N}$ such that $\tilde{a}_{j}=a_{j}$ for all $j \in N \backslash\{i\}$.

Based on Definitions 4.1 and 4.2 we can now extend the usual definition of a swing in order to include the distinction between weak and strong swings. We do this by fully counting all strong swings and counting weak swings only for a fraction $\epsilon \in[0,1]{ }^{14}$

Definition 4.4 Let $S \subseteq N$ and $a=\left(a_{i}\right)_{i \in S}$ be an action profile. For $\epsilon \in[0,1]$, the power ascription to $i \in S$, is given by

$$
\overline{P O W}_{i}^{\epsilon}(a, \Gamma)= \begin{cases}1 & \text { if } i \text { has a strong swing in a } \\ \epsilon & \text { if } i \text { has a weak swing in a } \\ 0 & \text { otherwise. }\end{cases}
$$

The power of an actor $i \in N$ in a decision-making body applying an DMM $\Gamma$ is measured by $i$ 's probability to have a swing, i.e., by summing-up over $i$ 's weighted power ascriptions $\overline{P O W}_{i}^{\epsilon}(a, \Gamma)$ for all feasible action profiles $a$.

Definition 4.5 For $\epsilon \in[0,1]$, the power measure POW $W^{\epsilon}$ of a decision-making body applying an $D M M \Gamma$ is given by

$$
P O W_{i}^{\epsilon}(\Gamma)=\sum_{a_{i} \in \mathcal{A}_{i}^{N}} p(a, \Gamma) \overline{P O W}_{i}^{\epsilon}(a, \Gamma) \text { for each } i \in N .
$$

\footnotetext{
${ }^{14}$ Note that van den Brink and Steffen (2008) demonstrate that it is not necessary to specify the value of $\epsilon$ for binary DMMs.
} 
Now, in order to link the notion (and measurement) of power with the notions (and measurement) of success and satisfaction as defined in Section 3 we will make use of two different notions of $l u c k .{ }^{15}$

The first notion of luck is based on Barry (1980) and Dworkin (1981). Barry (1980) has introduced the notion luck in the context of studying power and defines it as (the probability) of 'getting what one wants even when one does nothing', i.e., if 'one does not act', where according to our understanding the word 'even' is just a careless rhetorical flourish. ${ }^{16}$ Barry's (1980) definition of luck, we will use the notion Barry luck to refer to this, is in line with Dworkin's (1981) notion of brute good luck which he distinguishes from good option luck. The latter type of luck is related to "deliberate and calculated gambles ... - whether someone gains or loses through accepting an isolated risk he or she should have anticipated", while brute luck is regarded to be "a matter of how risks fall out that are not in that sense deliberate gambles." 17

Let $S \subseteq N \backslash\{i\}, a=\left(a_{j}\right)_{j \in S}$ be an action profile not containing an action from actor $i \in N \backslash S$, and $k \in K(a)$ be a corresponding inclination profile containing inclination $k_{i} \in K_{i}$ for actor $i$. Then, we ascribe brute good luck (or Barry luck) to $i$ if, and only if, $i$ 's inclination $k_{i}$ corresponds with the collective outcome $o(a, \Gamma) \in O$, but $i$ did not act.

\footnotetext{
${ }^{15}$ Note that, as pointed out by Dowding (1991: 64) with respect to one of these notions, both notions must be carefully distinguished from what he call's personal identity luck, i.e., "the luck of being the particular person one happens to be" which is discussed by egalitarians (see, for instance, Roemer 1986 or Cohen 1989).

${ }^{16}$ For a critical discussion of the probability requirement in Barry's (1980) luck definition see Dowding (1991: 65, 1996: 52f). Moreover, we would like to point out an inconsistency in Barry's (1980) analysis. His definition of decisiveness being: "the difference between his success [making use of the IOC] and his luck. ... it represents the difference that it makes to his success if he tries." Hence, whenever an actor tries according to this definition it is decisive, i.e., if $i$ is a dummy actor and $i$ chooses an action, i.e., $i$ tries to get what it wants, $i$ would be decisive. However, this is not what is usually meant by the notion of decisiveness (see Footnote 11) and Barry (1980) himself later in his essay writes that decisiveness means to be 'critical', i.e., to have a swing. Now one might argue, that Barry (1980) has meant this and that a dummy actor by definition cannot 'try', but this would mean that the notion of a 'try' presupposes the ability to be successful with the 'try'. However, this contradicts also the very basic meaning of a 'try' as being just an attempt in order to achieve something, whether one has the ability to do so or not. Taking this criticism into account one could re-define Barry's (1980) definition of luck to be: 'getting what one wants if one does not try or if one tries without being critical with respect to the action profile in question'. Note that the second part of this definition is the definition of action luck as contained in Definition 4.8.

${ }^{17}$ Dworkin (1981) illustrates the difference between both types of luck by an example of bad luck: "If someone develops cancer in course of a normal life, and there is no particular decision to which we can point as a gamble risking the disease, then we will say that he has suffered brute bad luck. But if he smoked cigarettes heavily then we may prefer to say that he took an unsuccessful gamble", i.e., he has suffered bad option luck.
} 
Definition 4.6 Let $S \subseteq N \backslash\{i\}, a=\left(a_{j}\right)_{j \in S}$ be an action profile not containing an action from actor $i \in N \backslash S$, and $k \in K(a)$ be a corresponding inclination profile containing inclination $k_{i} \in K_{i}$ for actor $i$. Then, the brute good luck ascription to $i$, is given by

$$
\overline{B G L}_{i}(k, a, \Gamma)= \begin{cases}1 & \text { if } k_{i} \sim o(a, \Gamma) \\ 0 & \text { otherwise }\end{cases}
$$

Remark 4.7 Remark 2.6 implies that under the canonical set-up brute good luck does not exist as all actors are obliged to choose an action.

The second notion of luck which we call action luck is based on Laruelle and Valenciano $(2005,2008)$. Let $S \subseteq N$ and $a=\left(a_{j}\right)_{j \in S}$ be an action profile containing actions $a_{j} \in A_{j}, j \in S$. Then, we ascribe action luck $(\overline{A L})$ to $i \in S$ if, and only if, (i) $i$ acts in action profile $a$ and (ii) $i$ 's chosen action $a_{i}$ corresponds with the collective outcome $o(a, \Gamma) \in O$, but $i$ has no swing in $a$.

Definition 4.8 Let $S \subseteq N$ and $a=\left(a_{j}\right)_{j \in S}$ be an action profile containing actions $a_{j} \in A_{j}$, $j \in S$. Then, the action luck ascription to $i \in S$, is given by

$$
\overline{A L}_{i}(a, \Gamma)= \begin{cases}1 & \text { if } a_{i} \sim o(a, \Gamma), \text { but } i \text { has no swing in a } \\ 0 & \text { otherwise. }\end{cases}
$$

Remark 4.9 Note that Laruelle and Valenciano $(2005,2008)$ mistakenly claim that their notion of luck (which we called action luck) is the notion of luck which has been proposed by Barry (1980).

Based on definitions 4.6 and 4.8 we can now formulate corresponding brute good luck and action luck measures. The brute good luck of an actor $i \in N$ in a decision-making body applying an DMM $\Gamma$ is measured by $i$ 's probability to have brute good luck, i.e., by summing-up over $i$ 's weighted brute good luck ascriptions $\overline{B G L}_{i}(k, a, \Gamma)$ for all feasible action profiles $a$ where $i$ does not act.

Definition 4.10 The brute good luck measure BGL of a decision-making body applying an $D M M \Gamma$ is given by

$$
B G L_{i}(\Gamma)=\sum_{a \in \mathcal{A}^{N} \backslash \mathcal{A}_{i}^{N}} p(a, \Gamma) \sum_{k \in K(a)} \tilde{p}(k, a, \Gamma) \overline{B G L}_{i}(k, a, \Gamma) \text { for each } i \in N
$$

The action luck of an actor $i \in N$ in a decision-making body applying an DMM $\Gamma$ is measured by $i$ 's probability to have action luck, i.e., by summing-up over $i$ 's weighted action luck ascriptions $\overline{A L}_{i}(a, \Gamma)$ for all feasible action profiles $a$ where $i$ does act. 
Definition 4.11 The action luck measure AL of a decision-making body applying an DMM $\Gamma$ is given by

$$
A L_{i}(\Gamma)=\sum_{a \in \mathcal{A}_{i}^{N}} p(a, \Gamma) \overline{A L}_{i}(a, \Gamma) \quad \text { for each } i \in N .
$$

Making use of the notions of brute good luck and action luck we can now state that 'satisfaction $=$ success + brute good luck' and that 'success $=$ power + action luck', which yields that 'satisfaction $=$ power + action luck + brute good luck'. The second and third equality only hold if we count weak swings and strong swings equally (i.e., if we take $\epsilon=1) .{ }^{18}$ For the case $\epsilon=1$ we shortly denote $\overline{P O W}_{i}(a, \Gamma)=\overline{P O W}_{i}^{1}(a, \Gamma)$ and $P O W_{i}(\Gamma)=P O W_{i}^{1}(\Gamma)$.

Proposition 4.12 For a decision-making body applying an DMM $\Gamma$ the following general equations hold:

(i) $S A T(\Gamma)=S U C(\Gamma)+B G L(\Gamma)$

(ii) $S U C(\Gamma)=P O W(\Gamma)+A L(\Gamma)$

(iii) $S A T(\Gamma)=P O W(\Gamma)+A L(\Gamma)+B G L(\Gamma)$

\section{PROOF}

(i) For all $i \in N$, we have

$$
\begin{aligned}
& S U C_{i}(\Gamma)+B G L_{i}(\Gamma) \\
& =\sum_{a \in \mathcal{A}_{i}^{N}} p(a, \Gamma) \overline{S U C}_{i}(a, \Gamma)+\sum_{a \in \mathcal{A}^{N} \backslash \mathcal{A}_{i}^{N}} p(a, \Gamma) \sum_{k \in K(a)} \tilde{p}(k, a, \Gamma) \overline{B G L}_{i}(k, a, \Gamma) \\
& =\sum_{\substack{a \in \mathcal{A}_{i}^{N} \\
a_{i} \sim o(a, \Gamma)}} p(a, \Gamma)+\sum_{\substack{a \in \mathcal{A}^{N} \backslash \mathcal{A}_{i}^{N} \\
k_{i} \sim o(a, \Gamma)}} p(a, \Gamma) \sum_{\substack{k \in K(a) \\
k_{i}}} \tilde{p}(k, a, \Gamma) \\
& =\sum_{a \in \mathcal{A}^{N}} p(a, \Gamma) \sum_{\substack{k \in K(a) \\
k_{i} \sim o(a, \Gamma)}} \tilde{p}(k, a, \Gamma) \\
& =\sum_{a \in \mathcal{A}^{N}} p(a, \Gamma) \sum_{k \in K(a)} \tilde{p}(k, a, \Gamma) \overline{S A T}_{i}(k, \Gamma)=S A T_{i}(\Gamma),
\end{aligned}
$$

\footnotetext{
${ }^{18}$ In fact, it is also possible to allow for any $\epsilon \in[0,1]$ but in that case we also need to redefine action luck taking account of weak and strong swings. Since this paper focusses on the distinction between success and satisfaction, we will not do that.
} 
where the first equality follows by definition of $S U C$ and $B G L$, the second equality follows from the definitions of $\overline{S U C}$ and $\overline{B G L}$, the third equality follows since $k_{i} \sim a_{i}$ for all $a \in \mathcal{A}_{i}^{N}$ implies that

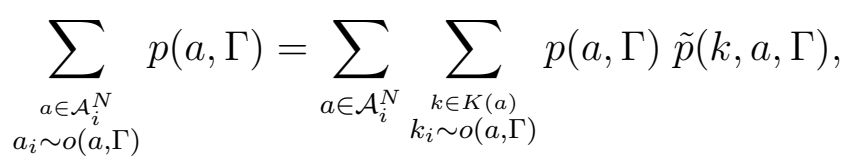

and the fourth and fifth equality follow from the definitions of $\overline{S A T}$ and $S A T$, respectively.

(ii) For all $i \in N$, we have

$$
\begin{aligned}
& P O W_{i}(\Gamma)+A L_{i}(\Gamma) \\
& =\sum_{a \in \mathcal{A}_{i}^{N}} p(a, \Gamma) \overline{P O W}_{i}(a, \Gamma)+\sum_{a \in \mathcal{A}_{i}^{N}} p(a, \Gamma) \overline{A L}_{i}(a, \Gamma)
\end{aligned}
$$

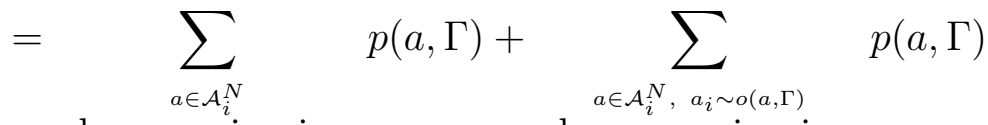

$$
\begin{aligned}
& i \text { has a swing in } a \quad i \text { has no swing in } a \\
& =\sum_{a \in \mathcal{A}_{i}^{N}} p(a, \Gamma)+\sum_{a \in \mathcal{A}_{i}^{N}} p(a, \Gamma)+\sum_{\substack{a \in \mathcal{A}_{i}^{N}, a_{i} \sim o(a, \Gamma) \\
\operatorname{los}^{\prime}}} p(a, \Gamma) \\
& i \text { has a swing in } a_{a_{i} \sim o(a, \Gamma)} \quad \quad \quad \quad \underset{\text { has a swing }}{a} \underset{a_{i} \chi_{0}(a, \Gamma)}{a} \quad i \text { has no swing in } a \\
& =\sum_{a \in \mathcal{A}_{i}^{N}} p(a, \Gamma)+\sum_{\substack{a \in \mathcal{A}_{i}^{N}, a_{i} \sim o(a, \Gamma) \\
i \operatorname{los}}} p(a, \Gamma) \\
& i \text { has a swing in } a \quad i \text { has no swing in } a \\
& a_{i} \sim o(a, \Gamma) \\
& =\sum_{\substack{a \in \mathcal{A}_{i}^{N} \\
a_{i} \sim o(a, \Gamma)}} p(a, \Gamma)=\sum_{a \in \mathcal{A}_{i}^{N}} p(a, \Gamma) \overline{S U C}_{i}(a, \Gamma)=S U C_{i}(\Gamma),
\end{aligned}
$$

where the first equality follows by definition of $P O W$ and $A L$, the second equality follows from the definitions of $\overline{P O W}$ and $\overline{A L}$, the fourth equality follows since $i$ has a swing in $a$ implies that $a_{i} \sim o(a, \Gamma)$, and the fifth and sixth equality follow from the definitions of $\overline{S U C}$ and $S U C$, respectively.

(iii) obviously follows from (i) and (ii).

If an DMM $\Gamma$ applied by a decision-making body is a canonical one, we obtain the following general equation 'satisfaction $=$ success $=$ power + action luck' which is quite close to Barry (1980), but contains the notion of luck as proposed by Laruelle and Valenciano (2005, 2008). 
Corollary 4.13 If the DMM $\Gamma$ applied by a decision-making body is a canonical one, we obtain the following general equation:

$$
S A T(\Gamma)=S U C(\Gamma)=P O W(\Gamma)+A L(\Gamma)
$$

\section{ProOF}

This follows since in the canonical set-up all actors act, and thus (i) an actor has success if, and only if, it has satisfaction and (ii) there is no brute good luck.

Remark 4.14 Corollary 4.13 reflects the content of Remark 3.10, i.e., that satisfaction and success coincide under the canonical set-up.

What remains to be demonstrated is that there exist applications for which brute good luck, which creates the wedge between satisfaction and success, plays an essential role. This is done in Section 5 by two examples of a sequential DMM.

\section{An Application: Sequential Decision-Making Mech- anisms}

Following van den Brink and Steffen $(2008,2012)$ let us assume a sequential 'one desk' DMM. Regarding the nature of the actor we have to distinguish between three types of actors: (i) 'bottom-', (ii) 'intermediate-', and (iii) 'top-actors'. 'Bottom actors' are those actors in the decision-making body who have a contact to the outside world and have the potential to receive new proposals, i.e., each decision-making body has at least one 'bottom-actor'. If there exists more than one bottom-actor we assume that a proposal enters the decision-making body via one of these actors with equal probability ('one desk' model). If a bottom-actor receives a proposal and does not support it by choosing the no-action, the proposal is regarded to be rejected by the decision-making body, i.e., the decision-making process is terminated. However, if the actor supports it by choosing the yes-action, it will be forwarded to the next actor in the decision-making process. This could be either an intermediate- or top-actor. It is an 'intermediate-actor' if regarding the consequences of its choice of action the same applies as for a bottom-actor, i.e., the difference between both types of actors lies just in the fact whether the actor has a contact to the outside world and can receive new proposals or not. A 'top-actor' is an actor who has no successors in the decision-making process. ${ }^{19}$ Hence, its choice of action leads always

\footnotetext{
${ }^{19}$ Note that the terminology successor-predecessor is opposite to the one as used in van den Brink and Steffen (2012). In van den Brink and Steffen (2012) both notions are used to refer to the positions of actors in a hierarchy, i.e., if actor $i$ directly dominates an actor $j$, we say that $i$ is a predecessor of $j$, and
} 
to a final collective decision on the proposal, i.e., a rejection of the proposal if it chooses the ' $n o-$ ' and an approval if it chooses the yes-action. Hence, top-actors are the only actors in the decision-making body who can finally enforce an approval of a proposal, while all actors have the ability to reject it (when they are allowed to choose an action). This implies that any sequential DMM in addition to at least one bottom-actor must also contain at least one top-actor, while the existence of intermediate-actors is not a necessary requirement.

Assumption 5.1 Let us add the following assumptions to those made under Assumption 2.1, where assumption 5 is now replaced by assumption 7:

7. All actors choose their action sequentially, where each actor $i \in N$ has not more than one chance to be involved in the decision-making, i.e., to choose its action.

8. New proposals entering the decision-making body can only be received by the bottomactor(s) being actors in positions with no predecessors in the decision-making process. This process continues then via the intermediate-actor(s) - if they exist - to the top$\operatorname{actor}(s)$.

9. A new proposal can only be received by one bottom-actor at the same time (One Desk Model).

10. The choice of the yes-action results (i) in a final approval if actor $i$ is the top-actor, i.e. if $i$ has no successor in the decision-making process, or (ii) in forwarding the proposal to one or more successors if $i$ is not the top-actor.

11. The choice of the no-action results in a final rejection of the proposal, if (i) actor $i$ is a bottom actor, or (ii) if for the actor who has forwarded the proposal to $i$ there is no other successor in the decision-making process left to ask for supporting the proposal whose individual support contains the potential of a final approval. If such other successor as in case (ii) exists it results in forwarding the proposal to this actor.

Remark 5.2 Note that under DMMs applying in addition to Assumption 2.1 also Assumption 5.1 - the latter contains a set of assumptions which are characteristic for hierarchical organizations (see van den Brink and Steffen 2008, 2012) - an actor $i$ can be excluded from the decision-making for two reasons: (i) if $i$ is an intermediate- or topactor the decision-making process could have already been terminated by another actor (which could be a bottom- or intermediate-actor) by choosing the no-action, or (ii) if the decision-making body contains more than one bottom-actor and the proposal has entered

that $j$ is a successor of $i$. In the present paper we make use of the same terminology to refer to actors in a sequential DMM, i.e., if an actor $i$ chooses its action after actor $j$ has made its choice of action, we say that actor $i$ is a successor of $j$, and that $j$ is a predecessor of $i$. 


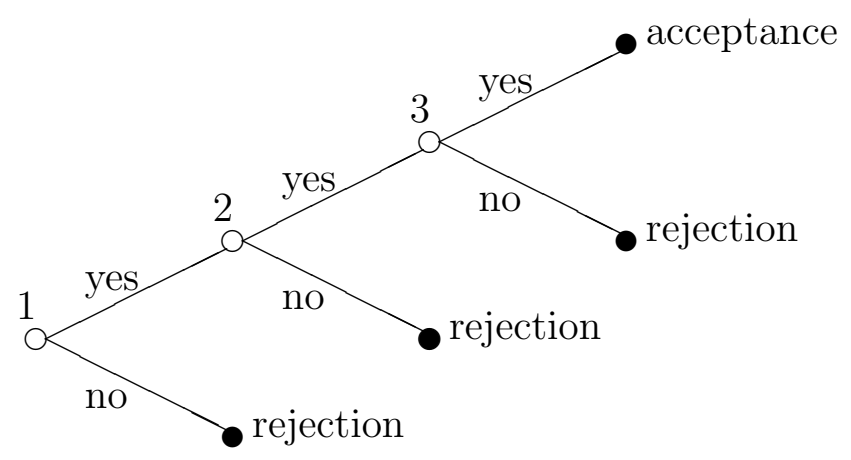

Figure 1: Extensive Game Form $\Gamma_{1}$

the decision-making body via a bottom-actor such that subsequent decision-making process does not include $i$ as an actor.

Clearly, in such sequential DMM's actors who are non-top-actors have strong and weak swings. A non-top-actor has a strong swing in action profiles where it is allowed to choose an action and where it chooses the yes-action and all its successors in the decision-making process choose the yes-action as well (leading to an approval of the proposal), while the proposal would be rejected if the actor chooses the no-action. It has a weak swing in every action profile where a non-top-actor is allowed to choose an action and where it chooses the no-action (leading to rejection of the proposal), while the proposal could be approved (if all its successors in the decision-making process who are allowed to choose an action choose the yes-action), but also could still be rejected (if there is at least one successor who is allowed to choose an action and chooses the no-action). A top-actor has two swings which are both strong.

Making use of Assumptions 2.1 and 5.1 we will now investigate two simple sequential DMMs in order to illustrate the relationship between satisfaction, success, and power and the usage of the related measures. ${ }^{20}$

Example 5.3 Let $\Gamma_{1}$ be a sequential DMM with $N=\{1,2,3\}$ where all actors choose their action sequentially in numerical ascending order, i.e., actor 1 is the unique bottom-, actor 2 is an intermediate-, and actor 3 is the unique top-actor. $\Gamma_{1}$ can be represented by the extensive game form as given by Figure 1. Our satisfaction, success, and power analysis is displayed by Table 2 assuming uniform probability distributions.

As it can be seen from Figure 1 and Table 2 under $\Gamma_{1}$ we have four action profiles $a$. In one of them actor 3 and in another one actors 2 and 3 are excluded from the decision-making

\footnotetext{
${ }^{20}$ Note that $\Gamma_{1}$ and $\Gamma_{2}$ in examples 5.3 and 5.4 , respectively, could be regarded as examples for DMMs in hierarchical organizations, where the structure of the hierarchy is a 'line' in case of $\Gamma_{1}$ and a 'star' in case of $\Gamma_{2}$ (see van den Brink and Steffen 2008, 2012).
} 


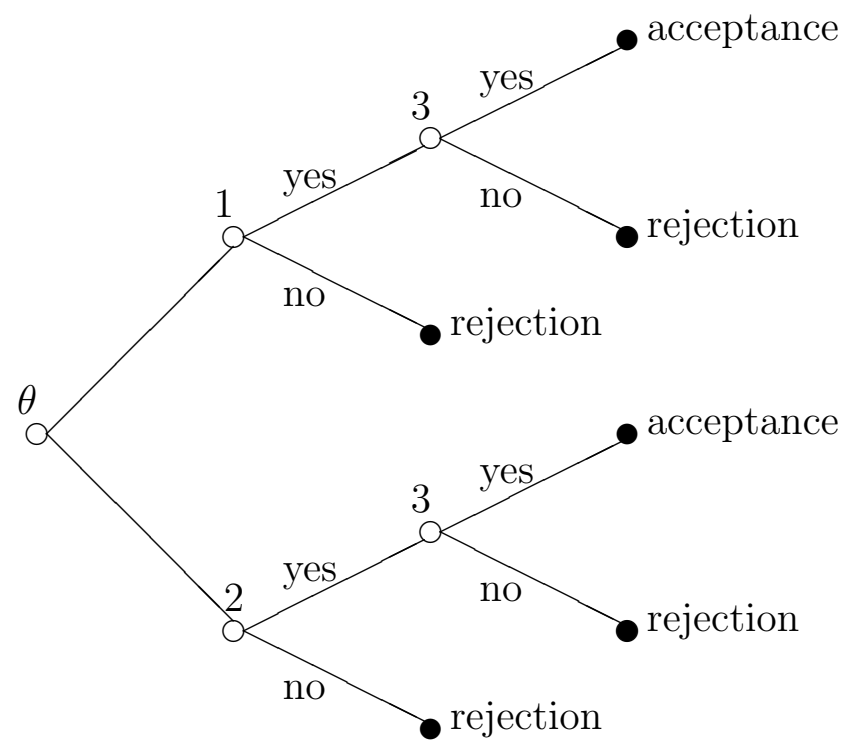

Figure 2: Extensive Game Form $\Gamma_{2}$

due to the fact that another actor, i.e., actor 2 in the former and actor 1 in the latter case, has chosen the no-action. Satisfaction of the excluded actors under these profiles is then a result of their brute good luck, only.

The next example includes also the second type of exclusion as explained in Remark 5.2 , i.e., exclusion due to the existence of more than one bottom-actor.

Example 5.4 Let $\Gamma_{2}$ be a sequential DMM with $N=\{1,2,3\}$. Let us assume that nature chooses with probability 0.5 whether bottom-actor 1 or 2 will receive a new proposal. After having received a proposal the bottom-actors can reject a proposal on their own, while for an approval both require the consent of top-actor $3 . \Gamma_{2}$ can be represented by the extensive game form as given by Figure 2. Our satisfaction, success, and power analysis is displayed by Table 3 assuming uniform probability distributions.

\section{Concluding Remarks}

The major purpose of this paper is to disentangle the relationship between satisfaction and success. Despite the fact that both notions are conceptually distinct they are mixedup in the voting power literature. We have pointed out that a potential explanation for this phenomenon is the fact, that both notions coincide under the canonical set-up which assumes a simultaneous DMM. Clarifying the relationship between both notions we found that the notion of luck requires an disentanglement as well. We illustrated the requirement for the disentanglements of all three notions by replacing the canonical simultaneous DMM 
by a sequential DMM, which allows that, under specific circumstances, some actors are excluded from the decision-making.

Now we would like to wind-up this paper with some remarks on the relationship between satisfaction and success if we allow for 'abstention' as a tertium quid. That is what Barry (1980) assumes when he illustrates his notion of luck under a simultaneous set-up. Hence, one might be tempted to argue that brute good luck can also exist under a simultaneous DMM if one allows for 'abstention' as a tertium quid. However, this would be mistaken as allowing for 'abstention' is nothing else than an extension of the action set: 'abstention' is an action an actor can choose in addition to the 'yes-' and no-action. In contrast to the 'non-action' of an excluded actor under a sequential DMM, an actor choosing the 'abstention-action' is not excluded from the decision-making by the definition of the decision-making procedure, but only due to its own choice of action not to opt in favor or against a proposal. Hence, we cannot ascribe brute good luck to such an actor. However, we also cannot ascribe action luck to such an actor as its choice of action does not correspond with the elements of the binary outcome set. What we require is a third notion of luck which we may call abstention luck being an additional component of satisfaction: 'satisfaction $=$ success + brute good luck + abstention luck'.

Regarding the ascription of abstention luck we have to distinguish between two principal cases if an actor chooses the 'abstention-action': (i) the actor has an inclination for one of the two elements of the outcome set, i.e., the actor either approves or rejects the proposal, but for certain reasons decides to choose the 'abstention-action', for instance, because the costs for performing the other actions are too high, or (ii) the actor is indifferent between the two elements of the outcome set. In the latter case we will always ascribe satisfaction to the actor, while in the former case satisfaction will only be ascribed if the collective outcome corresponds to its specific inclination for a unique element of the outcome set.

Finally, by making use of Barry's (1980) terminology we would like to point out that the difference between success and abstention luck can be characterized by the fact whether an actor 'has tried' or 'has not tried'. Remember that in Section 1 we noted that for his definition of success Barry (1980) adds the requirement that 'the actor has tried' which we used as a synonym for 'having chosen an action'. While both coincide under the canonical and our sequential set-up (see Footnote 3), this no longer holds if we allow for 'abstention' as a tertium quid. In this case we have to distinguish between two types of actions: (i) actions which imply that by their choice an actor 'tries' to obtain a specific collective outcome, e.g., the approval of a proposal by choosing the yes-action, and (ii) actions which imply that by their choice an actor 'does not try' to obtain a specific outcome. Actions of type (i) can lead to success, while actions of type (ii) can lead to 
abstention luck.

\section{References}

Banzhaf JF (1965), Weighted voting doesn't work: a mathematical analysis. Rutgers Law Review 19, 317-343.

Barry B (1980), Is it better to be powerful or lucky? Political Studies 28, 183-194, 338-352.

Braham M (2008), Social power and social causation: towards a formal synthesis. In: Braham M, Steffen F (eds) Power, freedom, and voting, Berlin, Heidelberg, Springer, pp 1-21.

Braham M, Steffen F (2002), Voting power in games with abstentions. In: Holler MJ, Kliemt H, Schmidtchen D, Streit ME (eds) Power and fairness, Tübingen, MohrSiebeck, pp 333-348.

Brams SJ, Lake M (1978), Power and satisfaction in a representative democracy. In: Ordeshook, PC (ed) Game Theory and Political Science, New York, New York University Press, pp 529-562.

Brink R van den, Rusinowska A, Steffen F (2011), Measuring power and satisfaction in societies with opinion leaders: dictator and new opinion leader properties. Homo Oeconomicus 28, 161-185.

Brink R van den, Steffen F (2008), Positional power in hierarchies. In: Braham M, Steffen F (eds) Power, freedom, and voting, Berlin, Heidelberg, Springer, pp 57-81.

Brink R van den, Steffen F (2012), Axiomatizations of a positional power score and measure for hierarchies. Public Choice 151, 757-787.

Cohen GA (1989), On the currency of egalitarian justice. Ethics, 99, 906-944.

Collins English Dictionary (2009), 10th ed., HarperCollins.

Dowding K (1991), Rational choice and political power. Aldershot, Edward Elagar.

Dowding K (1996), Power. Buckingham, Open University Press.

Dubey P, Shapley LS (1979), Mathematical properties of the Banzhaf power index. Mathematics of Operations Research, 4, 99-131. 
Dworkin R (1981), What is equality? Part 2: equality of resources. Philosophy of Public Affairs, 10, 283-345.

Felsenthal D, Machover M (1997), Ternary voting games. International Journal of Game Theory, 26, 335-351.

Felsenthal D, Machover M (1998), The Measurement of voting power: theory and practice, problems and paradoxes. London, Edward Elgar Publishers.

Felsenthal D, Machover M (2001), Models and reality: the curious case of the absent abstention. In: Holler MJ, Owen G (eds) Power indices and coalition formation, Dordrecht, Kluwer, pp 87-103.

Grabisch M, Rusinowska A (2010), A model of influence in a social network. Theory and Decision, 69, 69-96.

Holler MJ (1982), Forming Coalitions and measuring voting power. Political Studies, 30, 262-271.

Hosli MO, Machover M (2004), The Nice treaty and voting rules in the council: a reply to Moberg (2002). Journal of Common Market Studies, 42, 497-521.

Laruelle A, Martinez R, Valenciano F (2006), Success versus decisiveness: conceptual discussion and case study. Journal of Theoretical Politics, 18, 185-205.

Laruelle A, Valenciano F (2005), Assessing success and decisiveness in voting situations. Social Choice and Welfare, 24, 171-197.

Laruelle A, Valenciano F (2008), Voting and collective decision-making. Cambridge et al., Cambridge University Press.

Morriss P (1987/2002), Power: a philosophical analysis. Manchester, New York, Manchester University Press.

Nevison CH (1978), Structural power and satisfaction in simple games. Department of Mathematics, Colgate University, Hamilton, New York.

Nevison CH (1982), Structural satisfaction in simple games. Mathematical Social Sciences, 3, 397-401.

Penrose LS (1946), The elementary statistics of majority voting. Journal of the Royal Statistical Society, 109, 53-57. 
Rae D (1969), Decision rules and individual values in constitutional choice. American Political Science Review, 63, 40-56.

Roemer J (1986), Equality of resources implies equality of welfare. Quarterly Journal of Economics, 101, 751-784.

Rusinowska A, Swart H de, (2006), Generalizing and modifying the Hoede-Bakker index. In: Swart $\mathrm{H}$ de et al. (eds) Theory and applications of relational structures as knowledge instruments, Springer's Lecture Notes in Artificial Intelligence, LNAI 4342, Heidelberg, Springer, pp 60-88

Straffin PD (1977), Majority rule and general decision rules. Theory and Decision, 8, 351-360.

Straffin PD (1978), Probability models for power indices. In: Ordeshook PC (ed) Game theory and political science, New York, New York University Press, 477-510.

Straffin PD, Davis MD, Brams SJ (1982), Power and satisfaction in an ideologically divided voting body. In: Holler MJ (ed) Power, voting and voting power, Würzburg, Wien: Physica-Verlag, pp 239-255.

The American Heritage Dictionary of the English Language (2011), 5th ed, Houghton Mifflin Harcourt. 


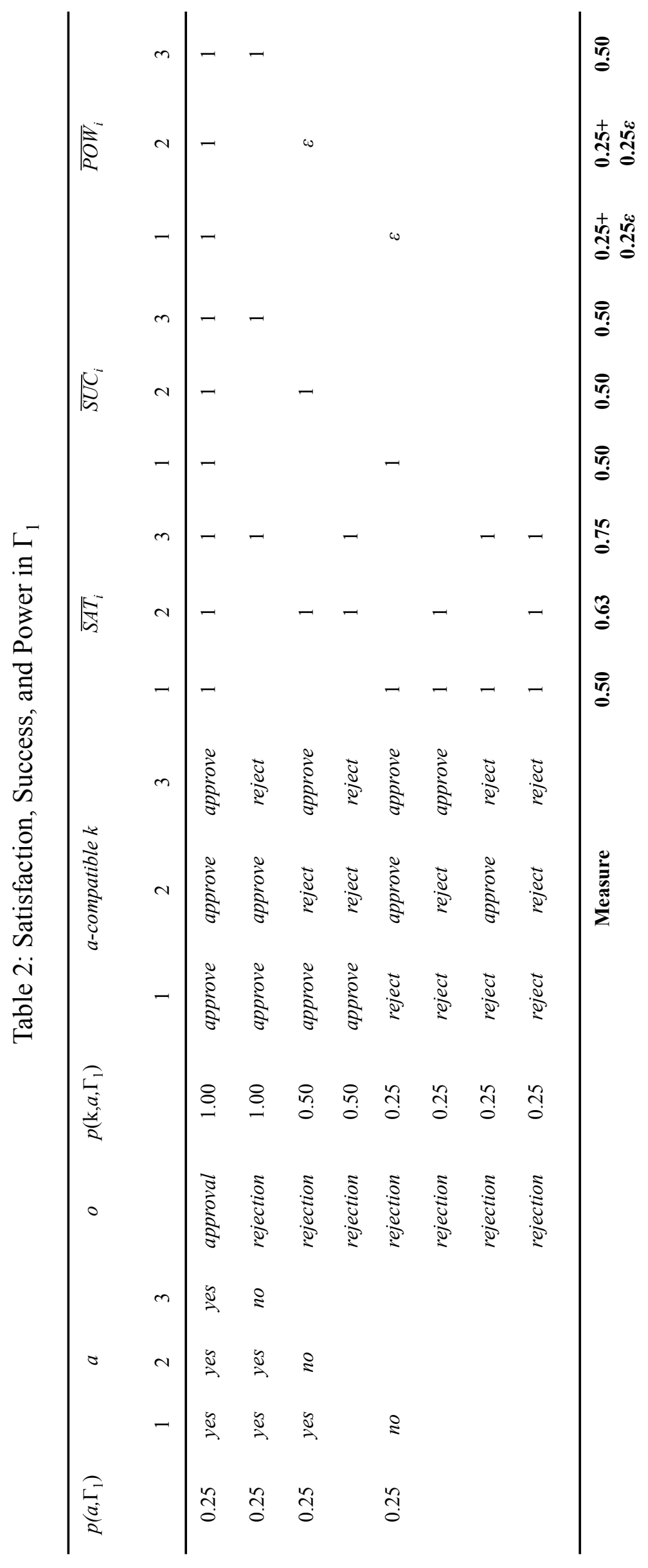




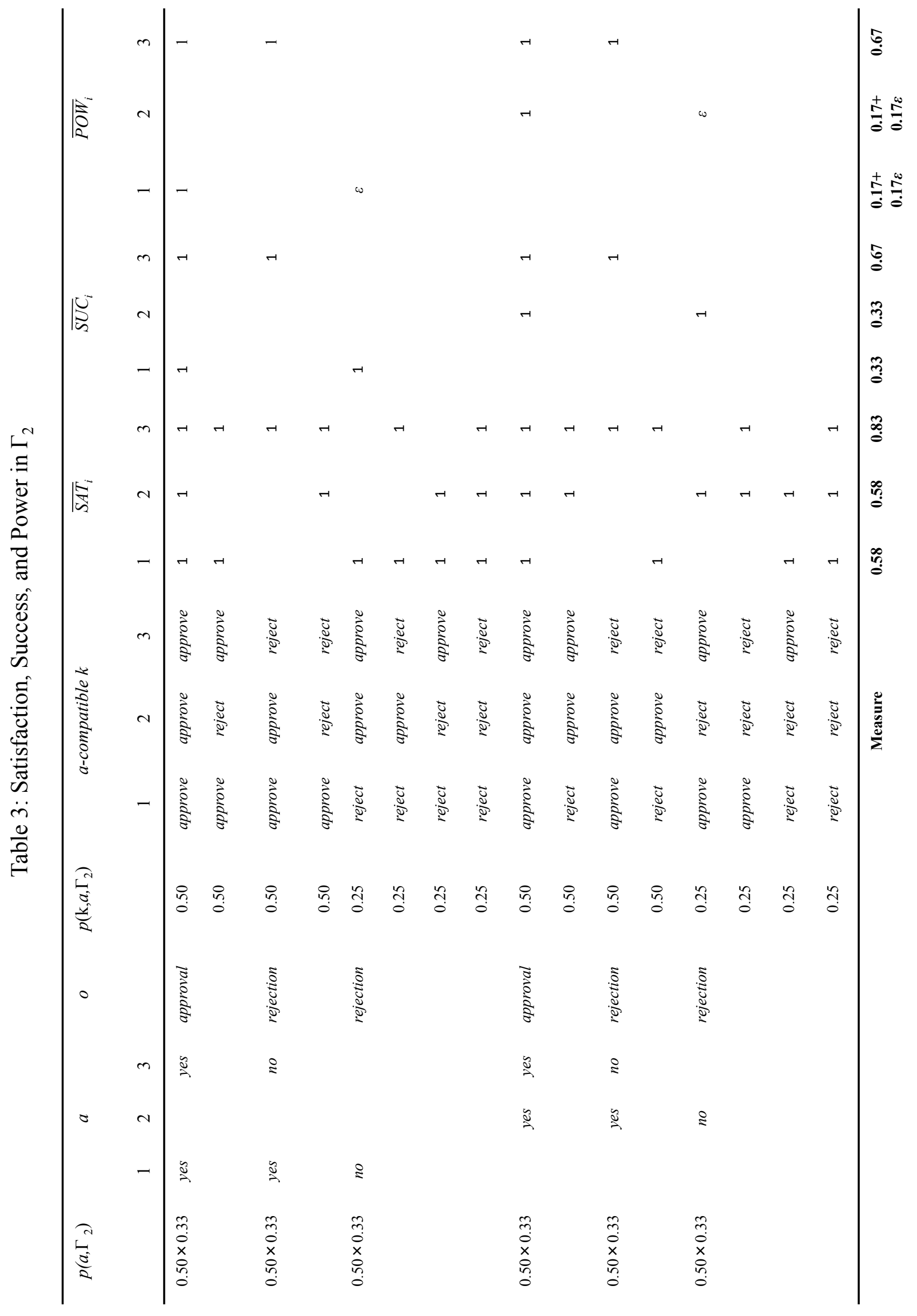

